\title{
From vision to action: meeting public health challenges in the Region
}

Ala Alwan ${ }^{1}$

Ministers of Health and high-level health officials will meet during the last week of October in Muscat, Oman, for the 60th WHO Regional Committee for the Eastern Mediterranean. The Regional Committee is WHO's decision-making body in the Eastern Mediterranean Region with representation from all Member States of the Region. The Committee will review the progress made in the Region in 2012 in various health programmes and consider strategies and ways forward to build on achievements and overcome challenges to move the countries of the Region forward to better health.

During the year 2012, Member States on several occasions endorsed the strategic priorities together with key strategic directions in public health for the Region. These include health system strengthening to achieve universal health coverage; the unfinished agenda in communicable diseases; maternal and child health, including reproductive health and nutrition; the prevention and control of noncommunicable diseases; and emergency preparedness and response. In this time, the Regional Office has worked tirelessly to respond to these challenges and to meet the expectations of Member States.

Accelerating progress towards universal health coverage by reforming national health systems is among the top priorities for the work of WHO in the Eastern Mediterranean Region. Achieving universal health coverage means ensuring access for all people to quality health services (including prevention, promotion, treatment and rehabilitation) without risk of financial hardship. To this end, WHO has been actively engaged in supporting Member States to: develop a comprehensive vision, evidence-based strategy and a well laid out road map; reform their entire health systems including health financing reform; adopt a multisectoral approach; and effectively monitor progress. Neither WHO nor Member States should underestimate the enormity of the challenge. Nevertheless with unwavering commitment and collective efforts it is achievable. Stronger health systems will improve the performance of all programmes and contribute to better health outcomes.

Member States have made commendable headway in tackling communicable diseases in the Region. Yet there is a significant unfinished agenda that remains and new challenges continue to emerge. The International Health Regulations lie at the heart of WHO's collaboration with its Member States on communicable diseases. The regulations comprise a core legal document that is binding on Member States who are expected to meet all requirements by 2014 . The emergence of the new coronavirus, Middle East respiratory syndrome (MERS-CoV), is a clear example of why we need the International Health Regulations. Despite the unequivocal commitment of the Regional Office, progress will not be possible unless the Member States reciprocate their obligation.

Among the most recalcitrant issues that confront us is the eradication of polio virus from the Region and the world. Two of the three countries in the world where the virus is still transmitted are in this Region. In May of this year, the World Health Assembly endorsed a new, comprehensive polio eradication and endgame strategic plan 2013-2018. There are major elements of this "endgame plan" that distinguish it from previous polio strategic plans. These include, among others, an urgent emphasis on improving immunization systems in key geographical areas; the introduction of new, affordable inactivated poliovirus vaccine; options for managing long-term poliovirus risks; strategies to address new threats, particularly insecurity in some endemic areas; and a concrete time line to complete the programme. Despite progress, challenges remain and new pockets of polio outbreaks keep emerging in different countries. It is unfortunate that these outbreaks can be mapped to those areas where the authority of the governments is weak and which are controlled by elements that impede initiatives to reach unvaccinated children. The complexity of the problem demands thinking "out of the box". Given that communities in the key polio-affected countries place a high level of trust in Islamic leaders and scholars, the Regional Office brought together scholars from these countries to exchange views and perceptions with international Islamic scholars to garner support in the push to eradicate polio.

Ten countries have been identified as high burden for maternal and child healthinthe Region. Withcurrent trends and unless action is scaled up, these countries will not achieve the targets 
of Millennium Development Goals 4 and 5. These countries require urgent support. WHO therefore resolved to muster commitment of concerned UN agencies and all countries of the Region to address this issue in the form of the Dubai Declaration for Saving the Lives of Mothers and Children. This was followed up by supporting countries to develop accelerated national plans for tackling maternal and child health problems. The closely related area of improving civil registration of births and deaths has also been taken up by the $\mathrm{WHO}$ as a priority. A preliminary situation analysis has been completed which will be followed up by the development of action plans, involving concerned stakeholders.

Noncommunicable diseases (NCDs) are taking a growing toll on the health of people of the Region. Last year the Regional Committee endorsed a regional framework for action on the commitments of Member States to implement the Political Declaration of the High-Level Meeting of the United
Nations General Assembly on the Prevention and Control of Noncommunicable Diseases. The numbers of people in the Region suffering from heart disease, diabetes and cancer are rising and will continue to rise as populations age. In parallel, the costs for individuals, families and governments of maintaining treatment for these chronic conditions are also rising. This Region is now beginning to accept the gravity of the situation and to address it, largely from the treatment aspect and not adequately from the viewpoint of prevention. We have high-impact cost-effective interventions to reduce exposure to risk factors for NCDs such as tobacco use, unhealthy diet and physical inactivity. These interventions, which represent "best buys" in public health terms, have been the focus of our work over the last year. We will continue to engage intensively with countries of the Region to help develop national programmes that address the surveillance, prevention and management of NCDs.
Emergencies in the Region seem almost to have become a way of life. Long-term conflict has long-term consequences for health. It drains health systems and it drains people, of life, energy and hope. Conflict, and the stress that accompanies it, affects mental health and is a serious risk factor noncommunicable diseases also. There is a major humanitarian situation within the Syrian Arab Republic and its neighbours as the numbers of displaced people and refugees continue to rise. This is having severe consequences for the health services of all the countries concerned and requires collective action and expression of solidarity to ensure that health care is afforded to the Syrian refugees and host communities.

The health mandate for the Region is huge and the expectations of the populations of the Region for better health are rising. To meet these expectations is a challenge to all Member States and to WHO. It requires the highest level of commitment, strong partnerships and concerted action. 